\title{
Formation of alcohols on ice surfaces
}

\author{
H. M. Cuppen, G. W. Fuchs, S. Ioppolo, S. E. Bisschop, K. I. Öberg, \\ E. F. van Dishoeck, and H. Linnartz
}

Raymond and Beverly Sackler Laboratory for Astrophysics

Leiden Observatory, Leiden University

P.O. Box 9513, 2300 RA Leiden, the Netherlands

email: cuppen@strw.leidenuniv.nl

\begin{abstract}
As the number of detections of complex molecules keeps increasing, answering the question about their formation becomes more pressing. Many of the saturated organic molecules are found to have a very low gas phase formation rate and are therefore thought to be formed on the icy surfaces of dust grains. In the Sackler Laboratory for Astrophysics we started a systematic study of the surface reaction routes that have been suggested over the years. Here we present the experimental results on the formation of methanol and ethanol by hydrogenation reactions of carbon monoxide and acetaldehyde ice. Computer simulations of the surface processes under similar conditions using the continuous-time random-walk Monte Carlo technique reveal some of the underlying physical processes. A better understanding of the physical conditions in which these molecules are formed can help in the interpretation of the observational results. The CO hydrogenation results will appear in detail in Fuchs et al. (2008). For more details on ethanol formation we refer to Bisschop et al. (2007).
\end{abstract}

Keywords. Astrochemistry, methods: laboratory, techniques: spectroscopic, molecular processes

\section{Introduction}

Surface processes play an important role in many astrophysical processes. Abundant molecules, like hydrogen, water and methanol, are formed mainly through surface reactions. Complete chemical networks on icy grains have been suggested where atom-bombardment by $\mathrm{H}, \mathrm{C}$, O, or $\mathrm{N}$ atoms leads to complex organic molecules as depicted in Figure 1. These reaction schemes as proposed by Tielens \& Hagen (1982) and Tielens \& Charnley (1997) are now in reach of experimental approaches and in the Sackler Laboratory for Astrophysics we have recently started a systematic study of this network by checking the different surface reactions and measuring the corresponding rates. This contribution summarizes the results on the formation of methanol and ethanol by hydrogenation reactions of carbon monoxide and acetaldehyde, indicated by the boxes in Figure 1. For hydrogenation studies of $\mathrm{CO}_{2}$ and $\mathrm{HCOOH}$, we refer to Bisschop et al. (2007).

\section{CO hydrogenation}

Laboratory studies in which CO ice is exposed to atomic hydrogen have been performed independently by two groups (Hiraoka et al. 1994, Watanabe \& Kouchi 2002). Hiraoka observed only formaldehyde formation, whereas Watanabe also found an effective methanol production. In a series of papers, these conflicting results have been discussed (Hiraoka et al. 2002, Watanabe et al. 2003, Watanabe et al. 2004) and the existing discrepancy 


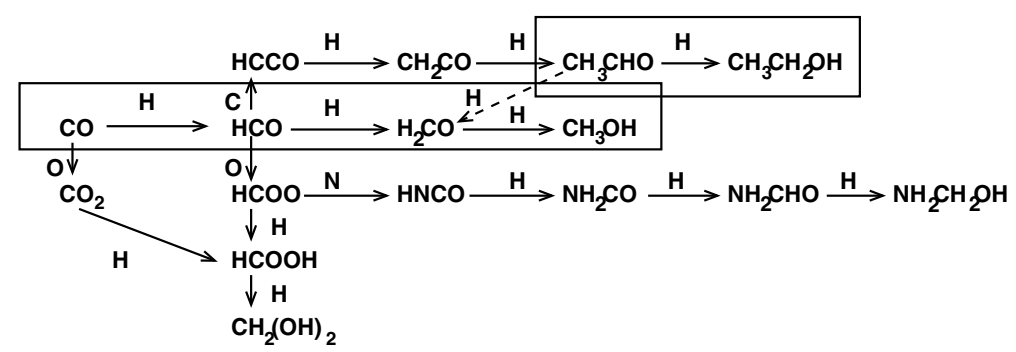

Figure 1. Surface reaction network (solid arrows) based on Tielens \& Charnley (1997). The boxes indicate the reaction scheme presented in the present work, the dash arrow the newly found route.

has been explained by different experimental conditions, most noticeable the adopted Hatom flux (Hidaka et al. 2004). An experimental verification, however, has been lacking so far. In order to verify the origin of the conflicting results, an additional systematic study has been performed focusing on the physical dependencies that may affect $\mathrm{CO}$ ice hydrogenation schemes, in particular: surface temperature and H-atom flux. For this purpose, a specialized UHV set-up has been used in which a CO ice layer of controllable thickness is deposited on a gold substrate. Typical thicknesses of 10 monolayers (ML) are used. These layers are then exposed to an atomic hydrogen beam obtained by a thermal cracking source. The decay of $\mathrm{CO}$ and the formation of the products formaldehyde and methanol are monitored in-situ by means of Reflection Absorption InfraRed Spectroscopy (RAIRS). After three hours of H-atom exposure, an additional analysis technique, Temperature Programmed Desorption (TPD), is applied in which the sample is heated and the desorption of the species is recorded as a function of temperature using a mass spectrometer. The symbols in Figure 2 show the evolution of $\mathrm{CO}, \mathrm{H}_{2} \mathrm{CO}$, and $\mathrm{CH}_{3} \mathrm{OH}$ as a function of fluence and time for a surface temperature of $12 \mathrm{~K}$ and a relatively high exposure of $5 \times 10^{13} \mathrm{H}$-atoms $\mathrm{cm}^{-2} \mathrm{~s}^{-1}$. Here clearly both $\mathrm{H}_{2} \mathrm{CO}$ and $\mathrm{CH}_{3} \mathrm{OH}$ are formed, in agreement with Watanabe et al. (2004). A comparible experiment is performed with a flux similar to the one applied by (Hiraoka et al. 1994). At this very low flux and fluence, $1 \times 10^{12} \mathrm{~cm}^{-2} \mathrm{~s}^{-1}$ and $1 \times 10^{16} \mathrm{~cm}^{-2}$, the formation of methanol cannot be confirmed by the RAIR spectra as its peak height is near the detection limit. However, the TPD spectrum shows a peak for mass 32 amu around the desorption temperature of methanol which is attributed to its formation. This leads to the conclusion that methanol is also formed at lower fluxes, although around the detection limit, and that the pricinple mechanism is not strongly dependent on the $\mathrm{H}$ flux.

\section{Interpretation by Monte Carlo simulations}

To interpret the results, a continuous-time, random-walk Monte Carlo simulation method was applied. This technique simulates a sequence of processes that can occur on the grain surface. These processes include hopping and desorption of the species and reactions between two species. It is a powerful tool to translate experimental data to interstellar conditions, since it can handle both the relatively high fluxes used in the laboratory and the low interstellar fluxes. It can also simulate a relatively large system over a long period of time, allowing for multiple processes to occur and to study their relative importance and interaction. In contrast with rate equation methods that are often applied, the method follows the individual atoms during a simulation, in this way one can consider the layering and topology of the system. For a detailed description of the 
method we refer to previous papers (Chang et al. 2005, Cuppen \& Herbst 2005, Cuppen \& Herbst 2007).

Here, two successive simulations are performed to simulate one experiment. First a CO layer is deposited starting from a bare surface and then during a second simulation this CO layer is exposed to hydrogen atoms. Input parameters for the simulations are hopping and reaction barriers and desorption energies. All processes except reactions are assumed to exhibit Arrhenius-like behavior where the barrier is crossed thermally. Some of the input parameters were varied in order to reproduce the experimental product evolutions. The solid lines in Figure 2 represent the simulated results that match the experiments. From our Monte Carlo analysis we can draw the following conclusions:

- the reaction rates show very little temperature dependence, indicating that tunneling through the reaction barrier is important,

- the production rate of methanol and formaldehyde decreases for increasing temperature, which is due to a higher hopping and desorption rate,

- the penetration depth of the hydrogen atoms into the $\mathrm{CO}$ ice is higher for higher surface temperatures, which is probably because of the higher mobility of the $\mathrm{CO}$ atoms in the ice.

The strong temperature dependence of the methanol formation rate may explain the large fluctuations in the astronomically observed methanol abundances.

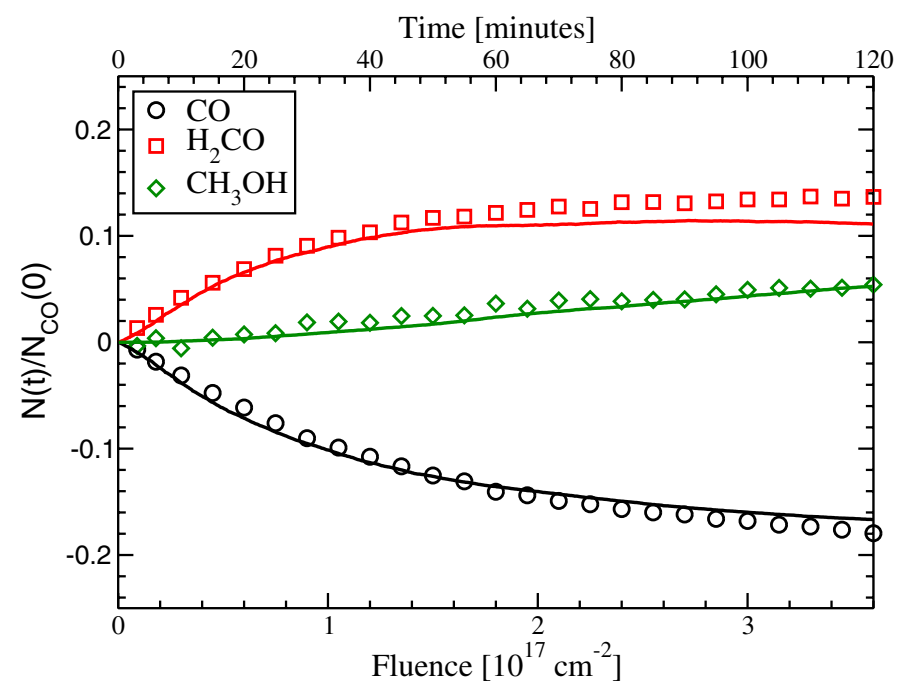

Figure 2. Evolution of $\mathrm{CO}, \mathrm{H}_{2} \mathrm{CO}$ and $\mathrm{CH}_{3} \mathrm{OH}$ with respect to the initial $\mathrm{CO}$-ice signal as a function of fluence and time. The symbols represent the experiment, the solid curves the results from the Monte Carlo simulations.

\section{Ethanol formation}

An additional series of experiments is performed in which acetaldehyde ice is exposed to H-atoms. According to the reaction scheme in Figure 1, ethanol should be formed in these experiments. However, in the RAIR spectra formaldehyde, methanol and methane were detected, suggesting that acetaldehyde breaks down into formaldehyde and methane upon hydrogen exposure. Formaldehyde is subsequently hydrogenated to methanol. No clear absorption is observed at $1050 \mathrm{~cm}^{-1}$, where the strongest $\mathrm{C}_{2} \mathrm{H}_{5} \mathrm{OH}$ band, the $\mathrm{C}=\mathrm{O}$ stretching mode, is expected. Since this frequency region is particularly problematic in our 


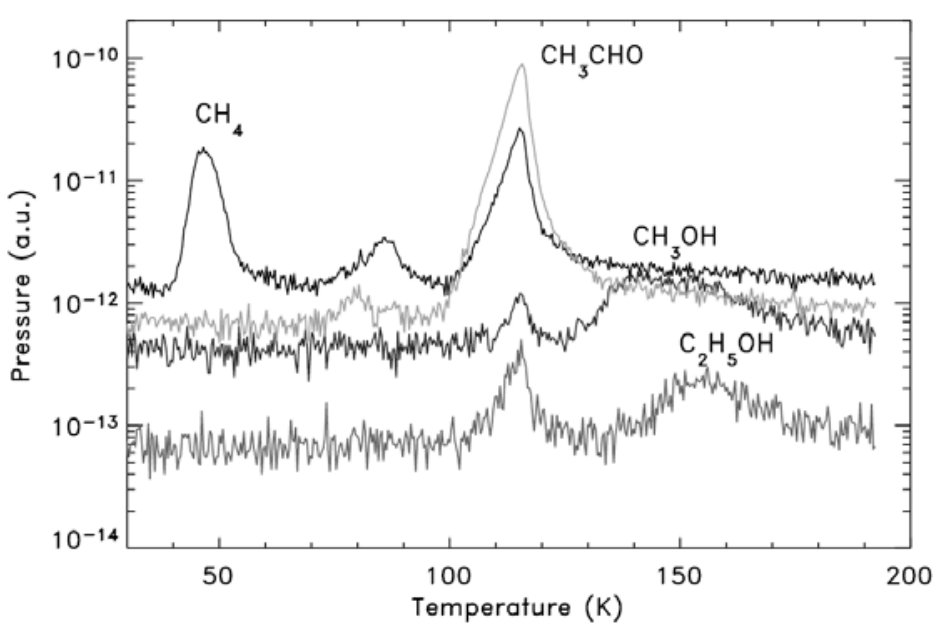

Figure 3. TPD spectra of acetaldehyde ice exposed to H-atoms $5 \times 10^{17} \mathrm{~cm}^{-2}$ at $14.5 \mathrm{~K}$. Methane, methanol and ethanol are formed. Taken from Bisschop et al. (2007).

detector, the detection sensitivity is low. Another strong band of ethanol is expected at $3.5 \mu \mathrm{m}$. Unfortunately, this feature overlaps with a number of methanol modes. Broad, weak features are indeed detected in this range, but due to the complexity of both $\mathrm{CH}_{3} \mathrm{OH}$ and $\mathrm{C}_{2} \mathrm{H}_{5} \mathrm{OH}$ absorptions and the relatively weak signal this cannot be used to determine whether methanol is present. Fortunately, more information can be obtained by inspection of the TPD spectra as shown in Figure 3. These corroborate the formation of $\mathrm{CH}_{4}, \mathrm{H}_{2} \mathrm{CO}$, and $\mathrm{CH}_{3} \mathrm{OH}$, which peak at $45 \mathrm{~K}, 100 \mathrm{~K}$, and $140 \mathrm{~K}$, respectively. In addition, a TPD desorption peak is located at $\sim 160 \mathrm{~K}$ for masses 45 and $46 \mathrm{amu}$. This is assigned to ethanol desorption based on a comparison with the TPD of pure nonbombarded acetaldehyde ices. In conclusion, a fraction of $\mathrm{CH}_{3} \mathrm{CHO}$, below the infrared detection limit of the $1050 \mathrm{~cm}^{-1}$ band, is converted to ethanol and a larger fraction forms formaldehyde, methanol, and methane. This experimentally confirms a pathway in the proposed reaction scheme by Tielens \& Charnley (1997) and allows us to add another pathway. The latter is indicated by the dashed arrow in Figure 1: from $\mathrm{CH}_{3} \mathrm{CHO}$ to $\mathrm{CH}_{2} \mathrm{O}$ under the influence of atomic hydrogen.

\section{References}

Bisschop, S. E., Fuchs, G. W., van Dishoeck, E. F., \& Linnartz, H. 2007, A\&A, 474, 1061

Chang, Q., Cuppen, H. M., \& Herbst, E. 2005, A\& $A, 434,599$

Cuppen, H. M. \& Herbst, E. 2005, MNRAS, 361, 565

Cuppen, H. M. \& Herbst, E. 2007, ApJ, 668, 294

Fuchs, G. W., Cuppen, H. M., Ioppolo, S., Bisschop, S. E., Andersson, S., van Dishoeck, E. F., \& Linnartz, H. 2008, AESA, submitted

Hidaka, H., Watanabe, N., Shiraki, T., Nagaoka, A., \& Kouchi, A. 2004, ApJ, 614, 1124

Hiraoka, K., Ohashi, N., Kihara, Y., Yamamoto, K., Sato, T., \& Yamashita, A. 1994, Chem. Phys. Lett., 229, 408

Hiraoka, K., Sato, T., Sato, S., Sogoshi, N., Yokoyama, T., Takashima, H., \& Kitagawa, S. 2002, ApJ, 577, 265

Tielens, A. G. G. M. \& Charnley, S. B. 1997, Origins of Life and Evolution of the Biosphere, 27,23

Tielens, A. G. G. M. \& Hagen, W. 1982, A\& $A$, 114, 245

Watanabe, N. \& Kouchi, A. 2002, ApJ (Letters), 571, L173 
Watanabe, N., Nagaoka, A., Shiraki, T., \& Kouchi, A. 2004, ApJ, 616, 638

Watanabe, N., Shiraki, T., \& Kouchi, A. 2003, ApJ (Letters), 588, L121

\section{Discussion}

Ceccarelli: Did you try to see what happens if you change the substrate? In particular, did you try to see whether you get a different result if your substrate is already ices, like water-ices?

Cuppen: We did some experiments with mixtures of ices. If you want to do this at low flux, I think you will really have a problem with detection. The work we've done this far was mostly in pure ices, but we did some work on mixtures of ices.

Ceccarelli: And did you find any differences?

CuPPEN: I will have to check and tell you later.

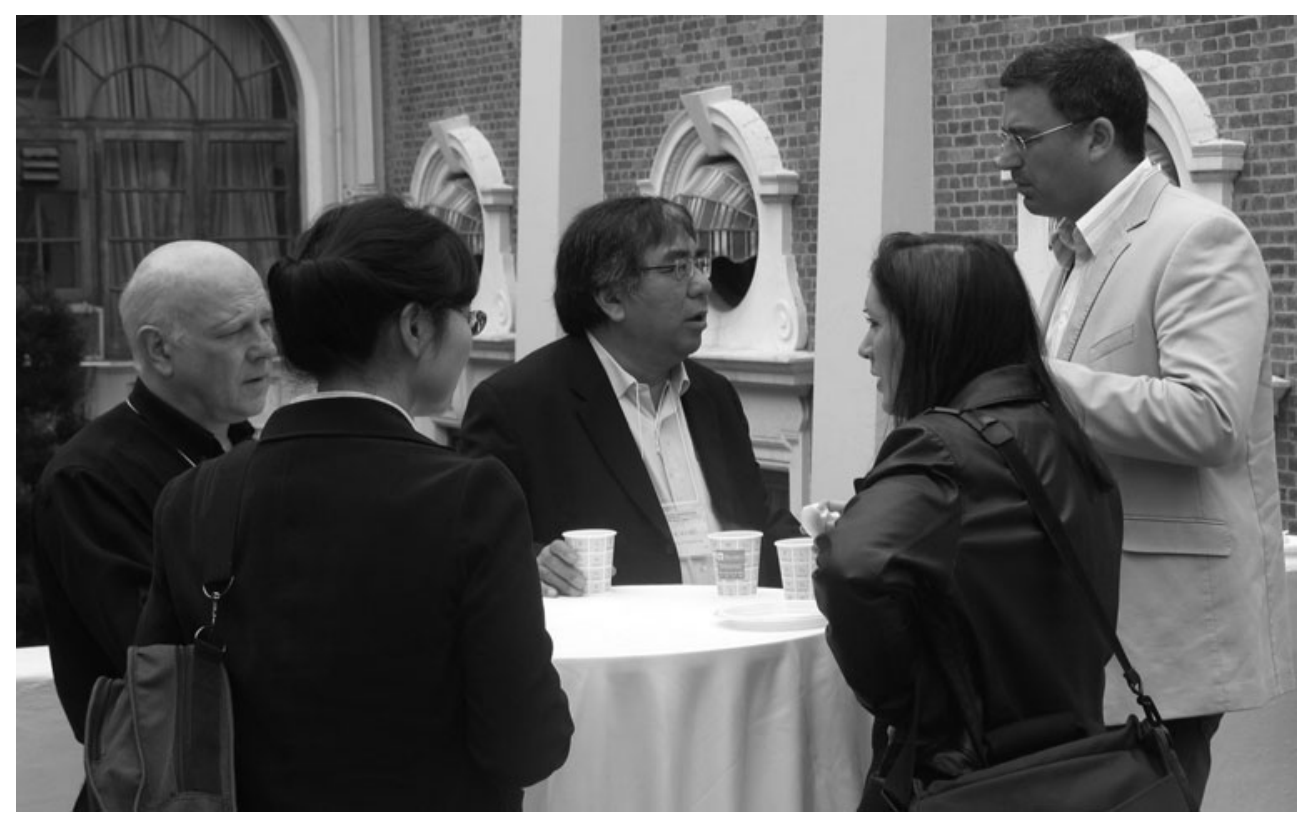

From left to right: Peter Sarre, In-Ok Song, Sun Kwok, Angela Speck, Steve Pointing (photo by Dale Cruikshank). 


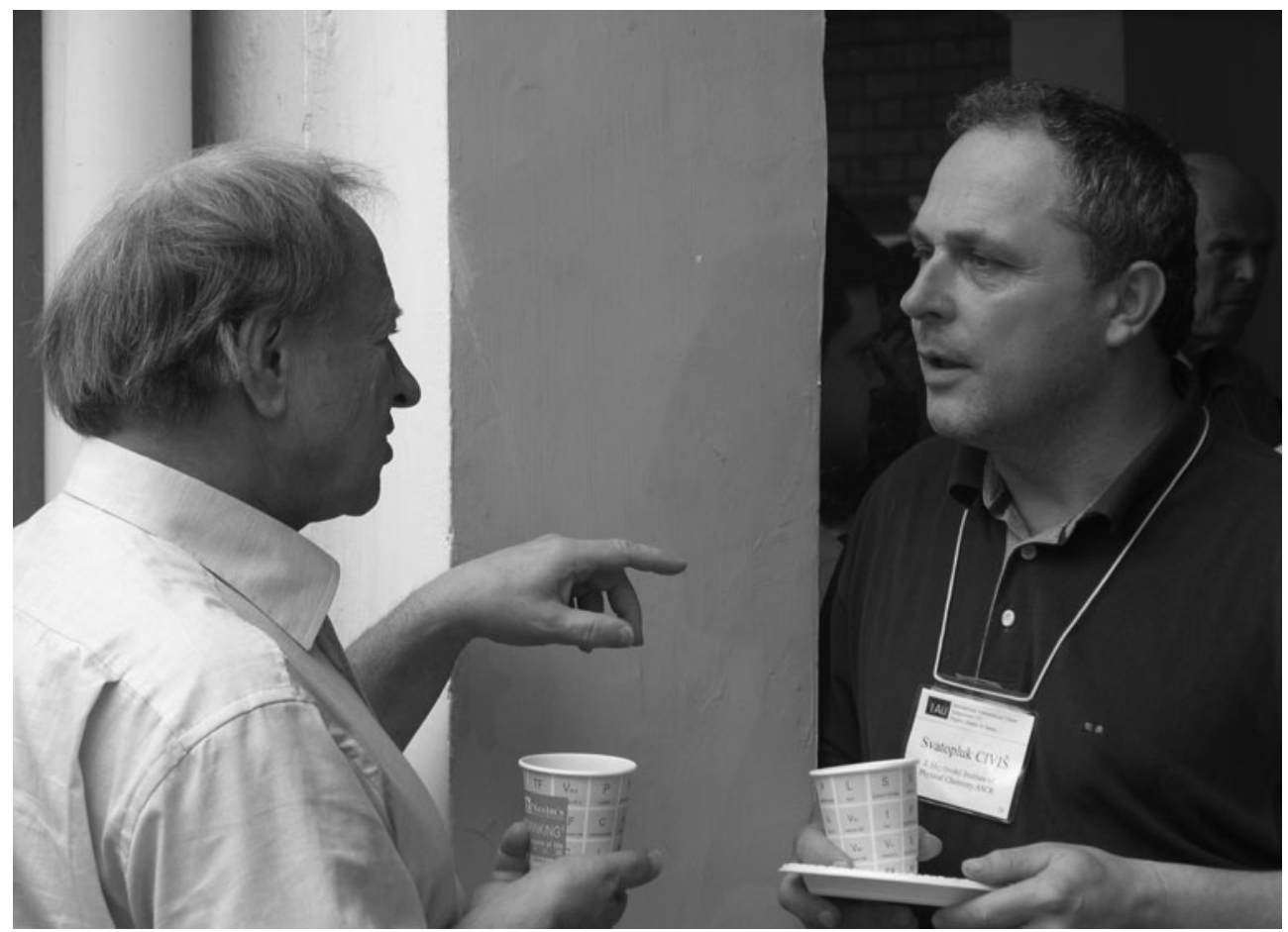

John Maier (left) and Svatopluk Civis (right). 